\title{
A computational biomarker of juvenile myoclonic epilepsy from resting- state MEG
}

\author{
Marinho A. Lopes ${ }^{\text {a,* }}$, Dominik Krzemiński ${ }^{a}$, Khalid Hamandi ${ }^{\text {a,b }}$, Krish D. Singh ${ }^{\text {a }}$, Naoki Masuda ${ }^{\text {c,d }}$, \\ John R. Terry ${ }^{\text {e,f,g }}$, Jiaxiang Zhang ${ }^{\text {a }}$ \\ ${ }^{a}$ Cardiff University Brain Research Imaging Centre, School of Psychology, Cardiff University, Cardiff CF24 4HQ United Kingdom \\ ${ }^{\mathrm{b}}$ The Welsh Epilepsy Unit, Department of Neurology, University Hospital of Wales, Cardiff CF14 4XW, United Kingdom \\ ${ }^{\mathrm{c}}$ Department of Mathematics, University at Buffalo, State University of New York, USA \\ d Computational and Data-Enabled Science and Engineering Program, University at Buffalo, State University of New York, USA \\ ${ }^{\mathrm{e}}$ EPSRC Centre for Predictive Modelling in Healthcare, University of Birmingham, Birmingham, United Kingdom \\ ${ }^{\mathrm{f}}$ Centre for Systems Modelling and Quantitative Biomedicine, University of Birmingham, Edgbaston, United Kingdom \\ ${ }^{\mathrm{g}}$ Institute for Metabolism and Systems Research, University of Birmingham, Edgbaston, United Kingdom
}

\section{A R T I C L E I N F O}

\section{Article history:}

Accepted 18 December 2020

Available online 04 February 2021

\section{Keywords:}

Epilepsy diagnosis

Juvenile myoclonic epilepsy

Biomarker

MEG

Functional connectivity

Phenomenological model

\section{H I G H L I G H T S}

- Computational modelling is combined with MEG to differentiate people with juvenile myoclonic epilepsy from healthy controls.

- Brain network ictogenicity (BNI) was found higher in people with juvenile myoclonic epilepsy relative to healthy controls.

- BNI's classification accuracy in our cohort was $73 \%$.

\begin{abstract}
A B S T R A C T
Objective: For people with idiopathic generalized epilepsy, functional networks derived from their resting-state scalp electrophysiological recordings have shown an inherent higher propensity to generate seizures than those from healthy controls when assessed using the concept of brain network ictogenicity (BNI). Herein we tested whether the BNI framework is applicable to resting-state magnetoencephalography (MEG) from people with juvenile myoclonic epilepsy (JME).

Methods: The BNI framework consists in deriving a functional network from apparently normal brain activity, placing a mathematical model of ictogenicity into the network and then computing how often such network generates seizures in silico. We considered data from 26 people with JME and 26 healthy controls.

Results: We found that resting-state MEG functional networks from people with JME are characterized by a higher propensity to generate seizures (i.e., higher BNI) than those from healthy controls. We found a classification accuracy of $73 \%$.

Conclusions: The BNI framework is applicable to MEG and was capable of differentiating people with epilepsy from healthy controls.

Significance: The BNI framework may be applied to resting-state MEG to aid in epilepsy diagnosis.

(c) 2021 International Federation of Clinical Neurophysiology. Published by Elsevier B.V. This is an open access article under the CC BY license (http://creativecommons.org/licenses/by/4.0/).
\end{abstract}

\section{Introduction}

Epilepsy is one of the most common neurological disorders with an estimated 5 million new diagnosis each year (WHO, 2019). The diagnosis of epilepsy is based on clinical history and supported by

\footnotetext{
* Corresponding author.

E-mail address: m.lopes@exeter.ac (M.A. Lopes).
}

clinical electroencephalography (EEG). The presence of interictal spikes in the routine scalp EEG recordings is one of the most valuable biomarkers of epilepsy (Pillai and Sperling, 2006). However, the presence of interictal epileptiform discharges (IED) in a routine EEG is low, ranging between 25 and 56\% (Smith, 2005; Benbadis et al., 2020). Furthermore, about $10 \%$ of people with epilepsy do not show IEDs even after repeated or prolonged EEG (Smith, 2005; Benbadis et al., 2020). On the other hand, specificity is also 
suboptimal, ranging between 78 and 98\% (Smith, 2005), which, for example, may delay the diagnosis of psychogenic nonepileptic attacks by 7 to 10 years (Benbadis, 2009).

The low sensitivity of IEDs results from IEDs being typically rare events. This may be a consequence of their sources being deep in the brain and/or the extent of cortex involved in epileptic activity being undetectable at the scalp surface (Pillai and Sperling, 2006). Consequently, much of the routine clinical EEG recording consists of brain activity that appears normal to visual inspection, which without other visible disturbances in background rhythms is considered non-informative. However, growing evidence suggests that such sections of interictal EEG without IEDs may be used to inform epilepsy diagnosis (e.g. Larsson and Kostov, 2005; Schmidt et al., 2016; Verhoeven et al., 2018). Larsson and Kostov (2005) showed that there is a shift in the peak of the alpha power towards lower frequencies in interictal EEG from people with both focal and generalized epilepsy. More recently, Abela et al. (2019) found that a slower alpha rhythm may be an indicator of seizure liability. Other studies have used graph theory to test whether functional networks derived from interictal EEG differ from EEG obtained from healthy controls. Functional networks are graphs, where nodes correspond to brain regions and connections are inferred from statistical dependencies (e.g. correlations) between brain signals from the regions. Such networks may represent the brain's functional coordination between regions (Park and Friston, 2013; Bassett and Sporns, 2017). It has been found that functional networks from people with epilepsy are more "regular" (i.e. higher path lengths between nodes) and deviate more from small-world structures than those found in healthy controls (Horstmann et al., 2010; Quraan et al., 2013). Functional network alterations inferred from resting-state EEG have also been used to differentiate children with focal epilepsy from healthy children (van Diessen et al., 2013, 2016). Furthermore, resting-state EEG functional networks from people with idiopathic generalized epilepsy (IGE) (also known as genetic generalised epilepsy) were shown to have more functional connections than healthy controls (Chowdhury et al., 2014). Functional networks inferred from interictal EEG from people with temporal lobe epilepsy have also been shown to differ from those from healthy controls (Coito et al., 2016).

All these studies show that functional networks based on apparently normal EEG may aid in the diagnosis of epilepsy. However, these studies lack mechanistic insights as to why such differences may be related to epilepsy. To build such understanding, we and others have proposed to use mathematical models of epilepsy to assess the functional networks and elucidate as to why a brain may be prone to generate seizures (Schmidt et al., 2014, 2016; Petkov et al., 2014; Lopes et al., 2019). In particular, resting-state EEG functional networks from people with IGE were shown to be more prone to support synchronization phenomena and the emergence of seizure-like activity than those from controls (Schmidt et al., 2014; Petkov et al., 2014). To quantify the differences, the concept of brain network ictogenicity (BNI) was introduced, i.e., a measure of how likely a functional network is of generating seizures in silico (Petkov et al., 2014).

For the BNI to be useful for diagnosing people with epilepsy from apparently normal brain activity, it relies on the assumption that the ability of a brain to generate seizures is an enduring feature that should be identifiable during interictal periods. It further assumes that such underlying closeness to seizures is captured by the properties of functional networks. Then, the capacity of a given functional network to generate seizures is assessed by estimating BNI through computer simulations that produce long-term activity from which the volume of epileptiform activity can be evaluated. People with epilepsy are therefore assumed to have resting-state functional networks that are more ictogenic, i.e., that have a higher propensity to generate seizures as estimated by the BNI, compared to healthy people (Schmidt et al., 2016).

Since MEG has the advantage, relative to EEG, of neuromagnetic fields being minimally perturbed by brain tissue, skull and scalp (Supek and Aine, 2016), we expect that MEG-derived functional networks would also be a candidate for the application of BNI. In the current study, we aimed to test whether the BNI concept can be applied to resting-state magnetoencephalography (MEG) data. In particular, we aimed to find whether MEG-derived BNI can differentiate juvenile myoclonic epilepsy (JME) from healthy controls. JME is recognised by the International League Against Epilepsy (ILAE) classification committee as a sub-syndrome of IGE, and makes up by far the largest proportion of IGE in terms of prevalence, alongside Juvenile Absence Epilepsy (JAE), Childhood Absence Epilepsy (CAE) and Epilepsy with Generalised Tonic Clonic Seizures on Awakening (GTCSA) (Engel, 2001; Scheffer et al., 2017). By applying the BNI framework to MEG, positive results would support the generalizability of the BNI across data modalities, over and above the previous EEG applications, and to a specific subsyndrome of IGE.

\section{Methods}

\subsection{Participants}

We used resting-state MEG data obtained from 26 people with JME and 26 healthy controls. The individuals with epilepsy were recruited from a specialist clinic for epilepsy at University Hospital of Wales in Cardiff, and the healthy individuals were volunteers who had no history of significant neurological or psychiatric disorders. The healthy group was age and gender matched to the epilepsy group. The age range in the epilepsy group was 17 to 47 , median 27 years, and in the control group was 18 to 48, median 27 years. There were 7 males in the epilepsy group and 7 males in the control group. Individuals in the epilepsy group had a number of different seizure types and were taking anti-seizure medications (see Krzemiński et al. (2020) and Routley et al. (2020) for more details about this dataset). Table 1 summarizes the clinical characteristics of the individuals with epilepsy. This study was approved by the South East Wales NHS ethics committee, Cardiff and Vale Research and Development committees, and Cardiff University School of Psychology Research Ethics Committee. Written informed consent was obtained from all participants.

\subsection{MEG acquisition and pre-processing}

MEG data were acquired using a 275-channel CTF radial gradiometer system (CTF System, Canada) at a sampling rate of $600 \mathrm{~Hz}$. We obtained approximately 5 minutes of MEG recordings per individual. The participants were instructed to sit steadily in the MEG chair with their eyes focused on a red dot on a grey background. Each individual also underwent a whole-brain T1weighted magnetic resonance imaging (MRI) acquired using a General Electric HDx 3 T MRI scanner and an 8-channel receiver head coil (GE Healthcare, Waukesha, WI) with an axial 3D fast spoiled gradient recalled sequence (echo time $3 \mathrm{~ms}$; repetition time $8 \mathrm{~ms}$; inversion time $450 \mathrm{~ms}$; flip angle $20^{\circ}$; acquisition matrix $256 \times 192 \times 172$; voxel size $1 \times 1 \times 1 \mathrm{~mm}$ ).

To assess the presence of artefacts and interictal spike wave discharges, the MEG data was divided into 2 s segments and each segment was visually inspected. Artefact-free segments were identified and re-concatenated for each individual. We thus obtained concatenated recordings with a variable length ranging from $204 \mathrm{~s}$ to $300 \mathrm{~s}$, and to avoid the potential impact of different 
Table 1

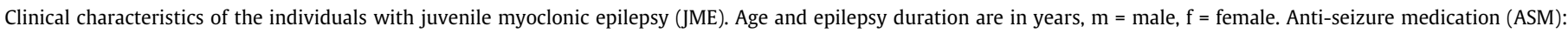

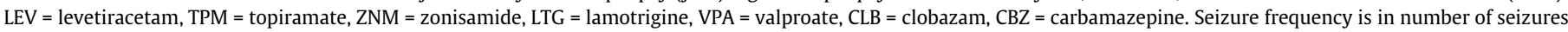

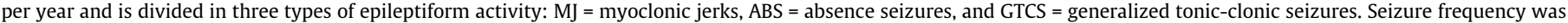
based on self-reporting at the time of scan and extrapolated to a number of seizures per year.

\begin{tabular}{|c|c|c|c|c|c|c|c|}
\hline \multirow[t]{2}{*}{ ID } & \multirow[t]{2}{*}{ Age } & \multirow[t]{2}{*}{ Gender } & \multirow[t]{2}{*}{ Epilepsy duration } & \multirow[t]{2}{*}{ ASM } & \multicolumn{3}{|c|}{ Seizure frequency } \\
\hline & & & & & MJ & ABS & GTCS \\
\hline JME1 & 17.8 & $\mathrm{f}$ & 2.8 & LEV & 12 & 365 & 3 \\
\hline JME2 & 31.3 & $\mathrm{f}$ & 18.3 & TPM, LEV & 12 & 12 & 1 \\
\hline JME3 & 27 & $\mathrm{f}$ & 19.0 & LEV, ZNM & 104 & 0 & 1 \\
\hline JME4 & 20.1 & $\mathrm{f}$ & 3.1 & LEV, TPM & 0 & 0 & 4 \\
\hline JME5 & 20.7 & $\mathrm{f}$ & 3.7 & LTG & 4 & 4 & 4 \\
\hline JME6 & 20.4 & $\mathrm{f}$ & 5.4 & LTG & 12 & 0 & 104 \\
\hline JME7 & 19.2 & $\mathrm{f}$ & 4.2 & LEV, ZNM & 12 & 12 & 12 \\
\hline JME8 & 20.9 & $\mathrm{f}$ & 12.9 & VPA, TPM & 104 & 36 & 4 \\
\hline JME9 & 35.3 & $\mathrm{f}$ & 23.3 & LTG, CLB & 2920 & 0 & 1 \\
\hline JME10 & 30.2 & $\mathrm{~m}$ & 16.2 & VPA & 52 & 52 & 4 \\
\hline JME11 & 23.7 & $\mathrm{~m}$ & 8.7 & VPA, LEV, CLB & 365 & 2 & 2 \\
\hline JME12 & 38.8 & $\mathrm{f}$ & 21.8 & VPA, ZNM & 365 & 365 & 104 \\
\hline JME13 & 22.2 & $\mathrm{~m}$ & 4.2 & VPA & 104 & 0 & 52 \\
\hline JME14 & 33.1 & $\mathrm{f}$ & 21.1 & LEV & 12 & 1 & 12 \\
\hline JME15 & 29.7 & $\mathrm{~m}$ & 14.7 & VPA & 12 & 0 & 0 \\
\hline JME16 & 25.7 & $\mathrm{f}$ & 10.7 & LEV, ZNM, LTG & 6 & 12 & 1 \\
\hline JME17 & 36 & $\mathrm{f}$ & 27.0 & LEV, TPM, CLB & 0 & 365 & 12 \\
\hline JME18 & 38.6 & $\mathrm{f}$ & 28.6 & VPA, LTG & 2 & 0 & 1 \\
\hline JME19 & 44.3 & $\mathrm{~m}$ & 29.3 & VPA, LEV & 365 & 365 & 2 \\
\hline JME20 & 47.7 & $\mathrm{f}$ & 40.7 & CBZ, LEV, CLB & 52 & 52 & 1 \\
\hline JME21 & 26.8 & $\mathrm{~m}$ & 8.8 & VPA & 1 & 0 & 0 \\
\hline JME22 & 22.3 & $\mathrm{f}$ & 10.3 & VPA & 6 & 0 & 1 \\
\hline JME23 & 38.7 & $\mathrm{f}$ & 24.7 & LEV, CLB & 0 & 0 & 1 \\
\hline JME24 & 18.9 & $\mathrm{f}$ & 3.9 & VPA & 0 & 0 & 1 \\
\hline JME25 & 31.1 & $\mathrm{f}$ & 18.1 & VPA & 1 & 0 & 0.2 \\
\hline JME26 & 22.7 & $\mathrm{~m}$ & 10.7 & LEV & 1 & 1 & 0.2 \\
\hline
\end{tabular}

recording lengths on our analysis, we only considered the first $200 \mathrm{~s}$ of each recording for every individual. The pre-processed data were then filtered in the alpha band $(8-13 \mathrm{~Hz}$ ) and downsampled to $250 \mathrm{~Hz}$. We focused on the alpha band because it has been shown to be the most informative for differentiating people with epilepsy from healthy controls (Schmidt et al., 2014, 2016).

\subsection{Source mapping from $M E G$}

To infer functional networks from the MEG data, we first mapped the data from the sensor space to the source space. The MEG sensors were co-registered with the structural MRI using the locations of the fiducial coils in the CTF software (MRIViewer and MRIConverter), and we obtained a volume conduction model from the MRI scan using a semi-realistic model (Nolte, 2003). To reconstruct the source signals, we used a linear constrained minimum variance (LCMV) beamformer on a $6-\mathrm{mm}$ template with a local-spheres forward model in Fieldtrip (Oostenveld et al., 2011; http://www.ru.nl/neuroimaging/fieldtrip). We mapped the source signals into the 90 brain regions of the Automated Anatomical Label (AAL) atlas (Hipp et al., 2012). For more details about these methods see our previous studies (Krzemiński et al., 2020, Routley et al., 2020).

\subsection{Functional networks}

We divided the 200-s-long source reconstructed MEG recordings into 10, non-overlapping, $20 \mathrm{~s}$ segments. The choice of segment length was motivated by previous studies that aimed to distinguish people with epilepsy from controls using restingstate scalp EEG (Schmidt et al., 2014, 2016). For each segment, we computed a functional network using the amplitude envelope correlation (AEC) with orthogonalized signals (Hipp et al., 2012) (see Supplementary Material S1 for more details). We selected this method because it has been shown to be a reliable measure of functional connectivity (Colclough et al., 2016). To remove spuri- ous connections, we generated 99 surrogates from the original MEG signals using the iterative amplitude-adjusted Fourier transform (IAAFT) with 10 iterations (Schreiber and Schmitz, 1996, 2000) (surrogates are randomized time series comparable to the original time series). We excluded connections if their weights did not exceed the 95\% significance level compared to the same connection weights as computed from the surrogates (Schmidt et al., 2014, 2016, Lopes et al., 2019). Using this method, we obtained 10 functional networks per individual.

\subsection{Mathematical model}

To study the inherent propensity of a MEG functional network to generate seizures, we placed a canonical mathematical model of ictogenicity at each network node, i.e. at each of the 90 brain regions represented in the functional network (Lopes et al., 2017, $2018,2019,2020)$. The activity of a network node was described by a phase oscillator, which could transit between two states: a 'resting state' at which the oscillator fluctuated close to a fixed stable phase and a 'seizure state' represented by a rotating phase (see Supplementary Material S2 for more details about the model). This canonical model has been shown to approximate the interaction between neural masses (Lopes et al., 2017).

\subsection{Brain network ictogenicity}

The mathematical model allowed us to generate synthetic brain activity which fluctuated between the resting and the seizure states. To quantify this activity, we used the BNI (Chowdhury et al., 2014; Petkov et al., 2014; Lopes et al., 2017, 2018, 2019, 2020), which is the average fraction of time that the network spent in the seizure state (see Supplementary Material S3 for more details). We interpret higher values of BNI as representing a higher inherent propensity of the brain to generate seizure activity. Thus, although we used resting-state MEG data to infer the functional networks, we assumed that the underlying brain states may differ 


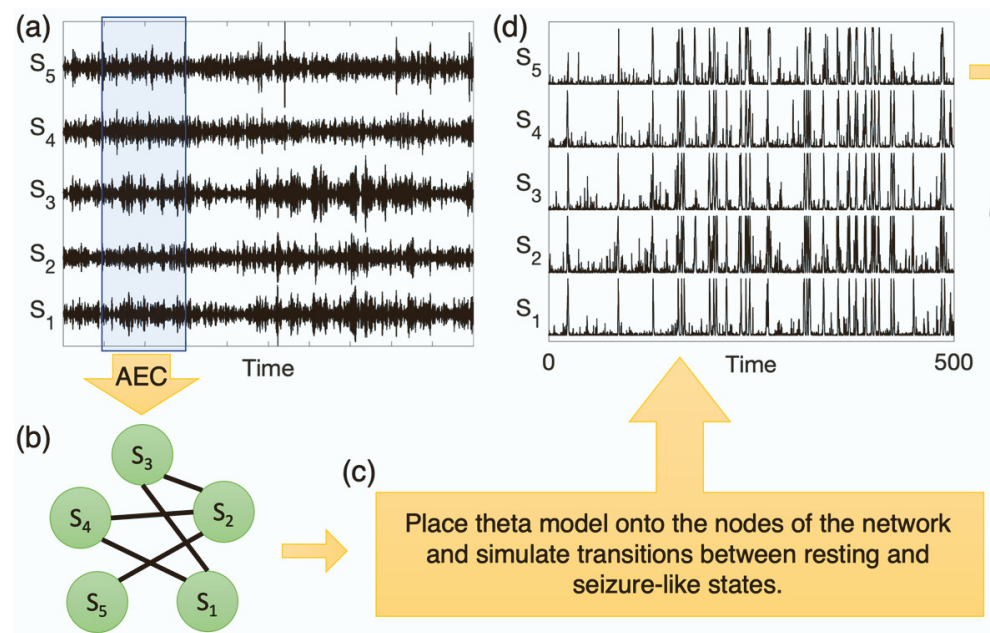

(e)

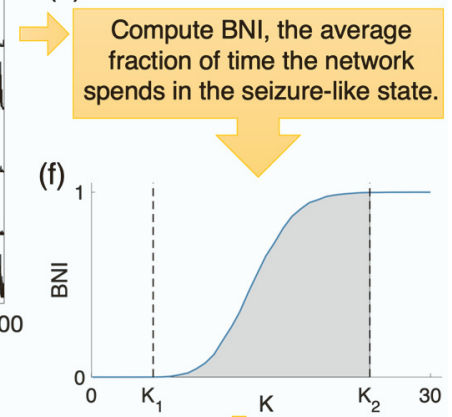

(g)

$\widehat{B N I}=$ area under the BNI curve

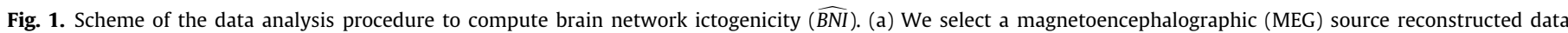

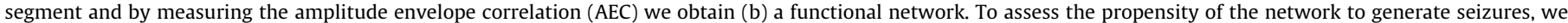

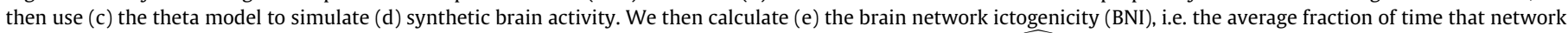

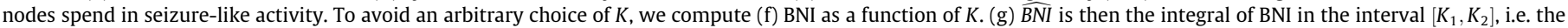
area under the BNI curve.

in their inherent propensity to generate seizures and this may be captured by our computational framework. We hypothesized that functional networks from JME individuals should be characterized by higher values of BNI than those from healthy individuals.

The simulated synthetic activity depends on a model parameter, the global scaling coupling $K$ (see Supplementary Material S2). Higher $K$ values imply stronger neuronal interactions between connected nodes, which in turn leads to higher BNI values. Hence, for a fair comparison of BNI between different functional networks, $K$ must be the same in all simulations. To avoid an arbitrary choice of $K$, we considered a redefinition of BNI (Lopes et al., 2018). This redefinition consists in computing BNI for a sufficiently large interval of $K$ values in order to capture the full variation of BNI from 0 to 1. Then we calculated $\widehat{B N I}$ as the integral of the $\mathrm{BNI}$ in this interval (see Supplementary Material S3). For a meaningful comparison between different functional networks, we used the same interval of $K$ for all simulations. This procedure has been shown to be robust (Lopes et al., 2018). Analogously to the BNI, a higher $\widehat{B N I}$ value corresponds to a higher propensity of a network to generate seizures. Fig. 1 summarizes the key steps of our method.

\subsection{Statistical methods}

We computed 10 functional networks per individual and therefore we obtained $10 \widehat{B N I}$ values per individual. We then calculated $\langle\widehat{B N I}\rangle$, the average of the $10 \widehat{B N I}$ values. Finally, we used the Mann-Whitney $U$ test to assess whether the median of $\langle\widehat{B N I}\rangle$ was higher in people with epilepsy than in the healthy controls.

\section{Results}

We considered resting-state MEG recordings from 26 people with JME and 26 healthy controls. To test whether $\widehat{B N I}$ was larger in individuals with JME than in healthy controls, we first built functional networks from MEG source reconstructed data, then we placed a mathematical model of ictogenicity into the network nodes and measured the networks' propensity to generate seizures in silico. Fig. 2(a) shows the $\widehat{B N I}$ for all individuals. Overall, individuals with JME had larger $\widehat{B N I}$ values than healthy controls
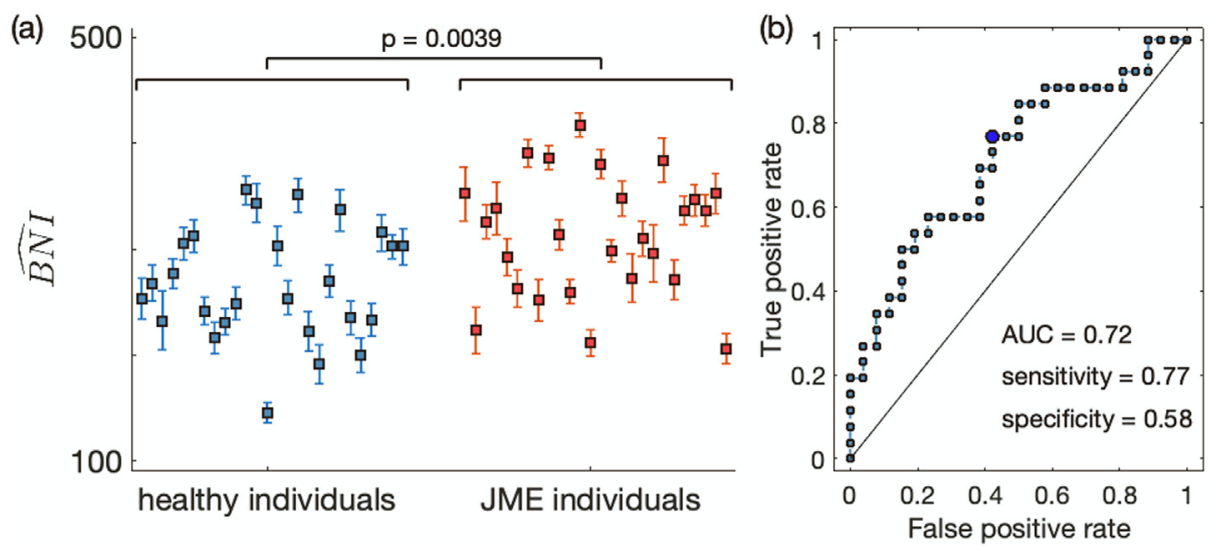

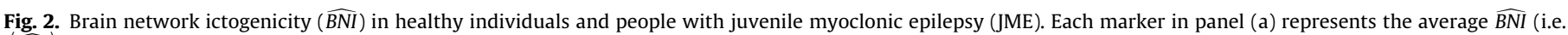

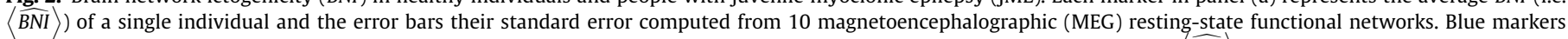

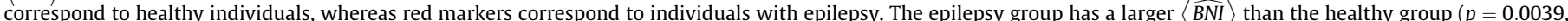

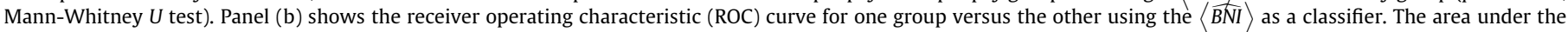
curve (AUC) is 0.72 and the circle identifies the optimal operating point of the ROC curve, for which the sensitivity is 0.77 , and the specificity is 0.58 . 
( $p=0.0039$, Mann-Whitney $U$ test). This finding confirms our hypothesis that resting-state functional networks from people with epilepsy have a higher propensity to generate seizures than those from healthy controls. Note also that for each individual, we observed that $\widehat{B N I}$ had a small variance (i.e. the intraindividual BNI variability is smaller than the interindividual BNI variability), implying that $\widehat{B N I}$ was consistent across the 10 different MEG resting-state functional networks of each individual. We then tested whether $\widehat{B N I}$ could be used for individual classification as to whether individuals had epilepsy. Fig. 2(b) shows the receiver operating characteristic (ROC) curve. The area under the curve (AUC) was 0.72 , the sensitivity was 0.77 , and the specificity was 0.58 . The $\widehat{B N I}$ 's classification accuracy was $73 \%$.

The results in Fig. 2 may be confounded by a number of factors. Namely, epilepsy duration and seizure frequency may have an impact on the $\widehat{B N I}$ values. Figure $\mathrm{S} 1$ shows the $\widehat{B N I}$ versus these clinical characteristics in the JME group. From visual inspection, the figure suggests that while individuals with short epilepsy duration or low seizure frequency may exhibit both low and high $\widehat{B N I}$ values, individuals with relatively longer epilepsy duration (larger than 20 years) and higher seizure frequency (higher than 200 seizures per year) present high $\widehat{B N I}$ values. We also computed the Pearson's correlation between the $\widehat{B N I}$ values and the seizure frequency of each seizure type (myoclonic jerks, absence seizures, and generalized tonic-clonic seizures), as well as the total seizure frequency across the three types. The correlations were not significant ( $r<0.29$ and $p>0.15$ in all tests).

\section{Discussion}

To date, the BNI framework has proved to be valuable for epilepsy diagnosis using scalp EEG in IGE (Schmidt et al., 2014, 2016; Petkov et al., 2014), assessment of epilepsy surgery using intracranial EEG in focal epilepsy (Goodfellow et al., 2016; Laiou et al., 2019; Lopes et al., 2020, 2018, 2017), and epilepsy classification using scalp EEG (Lopes et al., 2019). Here we extended previous results, testing whether the concept of BNI could differentiate people with JME from age and gender matched healthy controls using resting-state MEG data. We found that the BNI is on average higher in the JME group than in the control group. We further found that, as a classifier, the BNI yields a sensitivity of 0.77 , a specificity of 0.58 , and an AUC of 0.72 . This classification performance is similar to previous results in classifying people with IGE using scalp EEG (Schmidt et al., 2016). However, it is worth noting that our results from people with JME may not be directly comparable to those in the study by Schmidt et al. that were based on an IGE cohort that would have included other sub-syndromes, JAE, CAE and GTCSA, in addition to JME. Nevertheless, if we assume the existence of common traits across the IGE spectrum (including JME), our findings may suggest the hypothesis that MEG and scalp EEG may yield similar diagnostic power through the BNI framework, despite MEG often being considered superior to EEG in recording reliable brain signals (Supek and Aine, 2016). More generally, our results further consolidate the validity and usefulness of quantifying resting-state functional networks using a mathematical model of seizure transitions to assess the propensity of the brain to generate seizures.

Resting-state MEG functional networks have been previously shown to be altered in people with epilepsy relative to healthy controls (van Dellen et al., 2012; Niso et al., 2015; Hsiao et al., 2015; Wu et al., 2017; Routley et al., 2020). For example, Niso et al. (2015) used 15 graph-theoretic measures to quantify resting-state MEG functional networks from people with frontal focal epilepsy, generalized epilepsy and healthy individuals. They found that functional networks from generalized epilepsy had greater efficiency and lower eccentricity than those from controls, whereas functional networks from frontal focal epilepsy exhibited only reduced eccentricity over fronto-temporal and central sensors relative to networks from controls. Furthermore, machine learning has been used to also differentiate people with epilepsy from controls (Soriano et al., 2017). Our study distinguishes from these studies by not only searching for differences between functional networks in health and disease, but instead test a specific mechanistic hypothesis that justifies the difference. Thus, our approach is more readily interpretable and may offer insight into why altered functional networks underlie epilepsy.

We acknowledge that our study has some limitations. First, in order to truly test how MEG-based predictions compare to scalp EEG-based predictions, we would need both MEG and EEG data collected from the same participants. Future work should assess whether predictions based on both data modalities would deliver equivalent individual classification. Second, people with JME were taking anti-seizure medication, which may have potentially reduced the BNI in some JME individuals, making them indistinguishable from healthy individuals. Future studies should consider newly diagnosed drug-naïve individuals. This may be particularly important to also control for the effect of epilepsy duration and seizure frequency on BNI. Our results suggest that individuals with longer epilepsy duration and higher seizure frequency were more likely to be characterized by high BNI. On one hand this is an expected observation, i.e. BNI should be higher for individuals more prone to seizures and also those for which a longer disease may have had an impact on resting-state functional connectivity. On the other hand, these were individuals for which diagnosis could be less challenging. Third, we focused our analysis on differentiation of people with JME from healthy controls. We therefore cannot exclude the possibility that our findings are specific to JME. More comprehensive datasets will be needed to explore whether our findings generalize to other types of epilepsy.

\section{Conclusions}

By extending the application of the BNI framework to MEG, our results demonstrate that the $\mathrm{BNI}$ can be useful to interrogate different data modalities beyond EEG. We showed that resting-state MEG from people with JME is characterized by higher BNI than that from healthy controls. Our results suggest that BNI applied to resting-state MEG may aid in the diagnosis of JME.

\section{Declaration of Competing Interest}

JT is co-founder and Director of Neuronostics.

\section{Acknowledgements}

ML gratefully acknowledges funding from Cardiff University's Wellcome Trust Institutional Strategic Support Fund (ISSF) [204824/Z/16/Z]. DK was supported by an EPSRC PhD studentship [grant number EP/N509449/1]. KH acknowledges support from the Health and Care Research Wales: Clinical Research Time Award and the Wales BRAIN Unit. JT acknowledges the financial support of the EPSRC via grant EP/N014391/2 and a Wellcome Trust Institutional Strategic Support Award (WT105618MA). JZ acknowledges the financial support of the European Research Council [grant number 716321]. KDS and $\mathrm{KH}$ acknowledge the support of the UK MEG MRC Partnership Grant (MRC/EPSRC, MR/ K005464/1) and a Wellcome Trust Strategic Award (104943/Z/14/ Z). 


\section{Appendix A. Supplementary data}

Supplementary data to this article can be found online at https://doi.org/10.1016/j.clinph.2020.12.021.

\section{References}

Abela E, Pawley AD, Tangwiriyasakul C, Yaakub SN, Chowdhury FA, Elwes RD, et al. Slower alpha rhythm associates with poorer seizure control in epilepsy. Ann. Clin. Transl. Neurol. 2019;6(2):333-43. https://doi.org/10.1002/acn3.710.

Bassett DS, Sporns O. Network neuroscience. Nat. Neurosci. 2017;20(3):353-64. https://doi.org/10.1038/nn.4502.

Benbadis S. The differential diagnosis of epilepsy: a critical review. Epilepsy Behav. 2009;15(1):15-21. https://doi.org/10.1016/i.yebeh.2009.02.024.

Benbadis SR, Beniczky S, Bertram E, Maclver S, Moshé SL. The role of EEG in patients with suspected epilepsy. Epileptic Disord. 2020;22(2):143-55. https://doi.org/ 10.1684/epd.2020.1151.

Chowdhury FA, Woldman W, FitzGerald TH, Elwes RD, Nashef L, Terry JR, et al. Revealing a brain network endophenotype in families with idiopathic generalised epilepsy. PLoS One 2014;9(10). https://doi.org/10.1371/journal. pone.0110136 e110136.

Colclough GL, Woolrich MW, Tewarie PK, Brookes MJ, Quinn AJ, Smith SM. How reliable are MEG resting-state connectivity metrics?. NeuroImage 2016;138:284-93. https://doi.org/10.1016/j.neuroimage.2016.05.070.

Coito A, Genetti M, Pittau F, Iannotti GR, Thomschewski A, Höller Y, et al. Altered directed functional connectivity in temporal lobe epilepsy in the absence of interictal spikes: a high density EEG study. Epilepsia 2016;57(3):402-11. https://doi.org/10.1111/epi.13308.

van Dellen E, Douw L, Hillebrand A, Ris-Hilgersom IH, Schoonheim MM, Baayen JC, et al. MEG network differences between low-and high-grade glioma related to epilepsy and cognition. PLoS One 2012;7(11). https://doi.org/10.1371/iournal. pone.0050122.

Engel Jr J. A proposed diagnostic scheme for people with epileptic seizures and with epilepsy: report of the ILAE Task Force on Classification and Terminology.

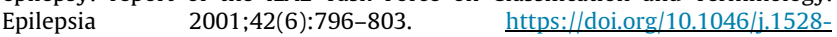
1157.2001.10401.x.

Goodfellow M, Rummel C, Abela E, Richardson M, Schindler K, Terry J. Estimation of brain network ictogenicity predicts outcome from epilepsy surgery. Sci. Rep. 2016;6(1):1-13. https://doi.org/10.1038/srep29215.

Hipp JF, Hawellek DJ, Corbetta M, Siegel M, Engel AK. Large-scale cortical correlation structure of spontaneous oscillatory activity. Nat. Neurosci. 2012;15(6):884. https://doi.org/10.1038/nn.3101.

Horstmann MT, Bialonski S, Noennig N, Mai H, Prusseit J, Wellmer J, et al. State dependent properties of epileptic brain networks: Comparative graphtheoretical analyses of simultaneously recorded EEG and MEG. Clin. Neurophysiol. 2010;121(2):172-85. https://doi.org/10.1016/ j.clinph.2009.10.013.

Hsiao FJ, Yu HY, Chen WT, Kwan SY, Chen C, Yen DJ, et al. Increased intrinsic connectivity of the default mode network in temporal lobe epilepsy: evidence from resting-state MEG recordings. PLoS One 2015;10(6). https://doi.org/ 10.1371/journal.pone.0128787.

Krzemiński D, Masuda N, Hamandi K, Singh KD, Routley B, Zhang J. Energy landscape of resting magnetoencephalography reveals fronto-parietal network impairments in epilepsy. Netw. Neurosci. 2020;1-23. https://doi.org/10.1162/ netn a 00125 .

Laiou P, Avramidis E, Lopes MA, Abela E, Müller M, Akman OE, et al. Quantification and selection of ictogenic zones in epilepsy surgery. Front. Neurol. 2019;10:1045. https://doi.org/10.3389/fneur.2019.01045.

Larsson PG, Kostov H. Lower frequency variability in the alpha activity in EEG among patients with epilepsy. Clin. Neurophysiol. 2005;116(11):2701-6. https://doi.org/10.1016/j.clinph.2005.07.019.

Lopes MA, Richardson MP, Abela E, Rummel C, Schindler K, Goodfellow M, et al. An optimal strategy for epilepsy surgery: Disruption of the rich-club?. PLoS Comput. Biol. 2017;13(8). https://doi.org/10.1371/journal.pcbi.1005637 e1005637.

Lopes MA, Richardson MP, Abela E, Rummel C, Schindler K, Goodfellow M, et al. Elevated ictal brain network ictogenicity enables prediction of optimal seizure control. Front. Neurol. 2018;9:98. https://doi.org/10.3389/fneur.2018.00098.
Lopes MA, Perani S, Yaakub SN, Richardson MP, Goodfellow M, Terry JR. Revealing epilepsy type using a computational analysis of interictal EEG. Sci. Rep. 2019;9 (1):1-10. https://doi.org/10.1038/s41598-019-46633-7.

Lopes MA, Junges L, Tait L, Terry JR, Abela E, Richardson MP, et al. Computational modelling in source space from scalp EEG to inform presurgical evaluation of epilepsy. Clin. Neurophysiol. 2020;131(1):225-34. https://doi.org/10.1016/ i.clinph.2019.10.027.

Niso, G., Carrasco, S., Gudín, M., Maestú, F., del-Pozo, F., \& Pereda, E. (2015). What graph theory actually tells us about resting state interictal MEG epileptic activity. NeuroImage: clinical, 8, 503-515. doi: 10.1016/j.nicl.2015.05.008

Nolte G. The magnetic lead field theorem in the quasi-static approximation and its use for magnetoencephalography forward calculation in realistic volume conductors. Phys. Med. Biol. 2003;48(22):3637. https://doi.org/10.1088/00319155/48/22/002.

Oostenveld R, Fries P, Maris E, Schoffelen JM. FieldTrip: open source software for advanced analysis of MEG, EEG, and invasive electrophysiological data. Comput. Intel. Neurosci. 2011;2011:1. https://doi.org/10.1155/2011/156869.

Park HJ, Friston K. Structural and functional brain networks: from connections to cognition. Science 2013;342(6158):1238411. https://doi.org/ $10.1126 /$ science. 1238411.

Petkov G, Goodfellow M, Richardson MP, Terry JR. A critical role for network structure in seizure onset: a computational modeling approach. Front. Neurol. 2014;5:261. https://doi.org/10.3389/fneur.2014.00261.

Pillai J, Sperling MR. Interictal EEG and the diagnosis of epilepsy. Epilepsia 2006;47:14-22. https://doi.org/10.1111/i.1528-1167.2006.00654.x.

Quraan MA, McCormick C, Cohn M, Valiante TA, McAndrews MP. Altered resting state brain dynamics in temporal lobe epilepsy can be observed in spectral power, functional connectivity and graph theory metrics. PLoS One 2013;8(7). https://doi.org/10.1371/iournal.pone.0068609.

Routley B, Shaw A, Muthukumaraswamy SD, Singh KD, Hamandi K. Juvenile myoclonic epilepsy shows increased posterior theta, and reduced sensorimotor beta resting connectivity. Epilepsy Res. 2020;106324. https://doi.org/10.1016/j. eplepsyres.2020.106324.

Scheffer IE, Berkovic S, Capovilla G, Connolly MB, French J, Guilhoto L, et al. ILAE classification of the epilepsies: position paper of the ILAE Commission for Classification and Terminology. Epilepsia 2017;58(4):512-21. https://doi.org/ 10.1111/epi.13709.

Schmidt H, Petkov G, Richardson MP, Terry JR. Dynamics on networks: the role of local dynamics and global networks on the emergence of hypersynchronous neural activity. PLoS Comput. Biol. 2014;10. https://doi.org/10.1371/journal. pcbi. 1003947 e1003947.

Schmidt H, Woldman W, Goodfellow M, Chowdhury FA, Koutroumanidis M, Jewell $\mathrm{S}$, et al. A computational biomarker of idiopathic generalized epilepsy from resting state EEG. Epilepsia 2016;57:e200-4. https://doi.org/10.1111/ epi.13481.

Schreiber T, Schmitz A. Improved surrogate data for nonlinearity tests. Phys. Rev. Lett. 1996;77(4):635. https://doi.org/10.1103/PhysRevLett.77.635.

Schreiber T, Schmitz A. Surrogate time series. Physica D 2000;142(3-4):346-82. https://doi.org/10.1016/S0167-2789(00)00043-9.

Smith SJM. EEG in the diagnosis, classification, and management of patients with epilepsy. J. Neurol. Neurosurg. Psychiatry 2005;76(suppl 2):ii2-ii7. https://doi. org/10.1136/innp.2005.069245.

Soriano MC, Niso G, Clements J, Ortín S, Carrasco S, Gudín M, et al. Automated detection of epileptic biomarkers in resting-state interictal MEG data. Front. Neuroinform. 2017;11:43. https://doi.org/10.3389/fninf.2017.00043.

Supek S, Aine CJ. Magnetoencephalography. Springer-Verlag, Berlin An. 2016. https://doi.org/10.1007/978-3-642-33045-2.

van Diessen E, Otte WM, Braun KP, Stam CJ, Jansen FE. Improved diagnosis in children with partial epilepsy using a multivariable prediction model based on EEG network characteristics. PLoS One 2013;8(4). https://doi.org/10.1371/ journal.pone.0059764.

van Diessen E, Otte WM, Stam CJ, Braun KP, Jansen FE. Electroencephalography based functional networks in newly diagnosed childhood epilepsies. Clin. $\begin{array}{lll}\text { Neurophysiol. } & \text { 2016;127(6):2325-32. https://doi.org/10.1016/ }\end{array}$ i.clinph.2016.03.015.

Verhoeven T, Coito A, Plomp G, Thomschewski A, Pittau F, Trinka E, et al. Automated diagnosis of temporal lobe epilepsy in the absence of interictal spikes. NeuroImage Clin. 2018;17:10-5. https://doi.org/10.1016/i.nicl.2017.09.021.

WHO (2019) https://www.who.int/news-room/fact-sheets/detail/epilepsy

Wu C, Xiang J, Jiang W, Huang S, Gao Y, Tang L, et al. Altered effective connectivity network in childhood absence epilepsy: a multi-frequency MEG study. Brain Topogr. 2017;30(5):673-84. https://doi.org/10.1007/s10548-017-0555-1. 